Revista de Filosofía y Teoría Política, n. ${ }^{\circ}$ 49, 2018, e022, ISSN 2314-2553

Universidad Nacional de La Plata.

Facultad de Humanidades y Ciencias de la Educación.

Departamento de Filosofía

\title{
Disputar la reforma, disputar la filosofía: Valoraciones y la Revista de Filosofía
}

\author{
María Carla Galfione \\ Universidad Nacional de Córdoba - CONICET, Argentina \\ carlagalfione@yahoo.com.ar
}

Cita sugerida: Galfione, M. C.(2018). Disputar la reforma, disputar la filosofía: Valoraciones y la Revista de Filosofía. Revista de Filosofía y Teoría Política, (49), e022. https://doi.org/10.24215/23142553e022 


\title{
Disputar la reforma, disputar la filosofía: Valoraciones y la Revista de Filosofía
}

\author{
Maria Carla Galfione \\ Universidad Nacional de Córdoba - CONICET, Argentina
}

\section{Resumen:}

La Reforma Universitaria puede ser considerada como el escenario propicio para el establecimiento de algunos presupuestos de la filosofía universitaria. Las definiciones que se operaban a comienzos de siglo pueden haber sido la base sobre la que se construyó el discurso del reformismo universitario. De uno u otro modo, ambos elementos se vinculan. En este artículo, nos aproximamos a la cuestión a partir de la lectura y la revisión de la propuesta editorial, filosófica y universitaria de la Revista de Filosofía. Cultura, ciencia, educación y de Valoraciones, dos publicaciones que se presentan como expresiones del reformismo universitario, al tiempo que intentan delimitar el campo filosófico.

Palabras clave: Filosofía universitaria, Reformismo universitario, Publicaciones periódicas, Debates intelectuales.

\section{Abstract:}

The university reform can be considered as the favorable scenario for the explanation of some supposed of the university philosophy. The definitions that were operated at the beginning of the century may have been the basis on which the discourse of university reformism was constructed. In one way or another, both elements are linked. In the article we approach the question from the reading and review of the editorial, philosophical and university proposal of the Revista de Filosofia. Cultura, ciencia, educación and Valoraciones. These are two publications that are presented as expressions of university reformism, while trying to delimit the philosophical field.

KEYWORDS: University philosophy, University Reformism, Journals, Intellectual debates.

En los años que mediaron entre los sucesos de junio de 1918 y la celebración de su décimo aniversario, reconocemos una invitación a pensar la relación entre las lecturas de la Reforma Universitaria y lo que se constituía como "discurso del reformismo", por una parte, y algunas definiciones de la filosofía universitaria que serían fundantes en la academia argentina, por la otra. Los años próximos a la Reforma formaban parte también del período en que se fundaban las bases de la filosofía, y ello supuso algunos debates importantes. Muchos de éstos coincidieron con los posicionamientos asumidos con respecto a la Universidad y su organización, y con respecto al conocimiento, sus definiciones y su rol. Pensar el rumbo que debía tomar la Universidad parecía ser, al mismo tiempo, sentar las bases de una filosofía. Las tareas que se consideraban propias del reformismo -y en muchos casos, aún pendientes-, fueron generalmente leídas como tareas de la filosofía, y derivaban en propuestas claras de lineamientos y disputas por la definición de la disciplina.

En lo que sigue, nos detendremos en los desarrollos que ofrecen Valoraciones y la Revista de Filosofía, dos publicaciones muy próximas, pero con diferencias importantes, y que permiten reconocer el modo en que fue estableciéndose ese estrecho vínculo entre las definiciones del reformismo y el debate en torno a la filosofía y su perfil en tanto disciplina académica. Ambas publicaciones pueden ser leídas como expresiones centrales del debate de entonces en torno a la filosofía. El sólo reconocimiento de sus directores (referentes y maestros, al mismo tiempo), Alejandro Korn y José Ingenieros, pone en evidencia a figuras centrales del reformismo que, al mismo tiempo, resultan ser protagonistas de los debates filosóficos del momento, sugiriendo una especie de desajuste que ha sido poco examinado hasta ahora.

El trabajo con revistas (lo advertimos brevemente, aunque no ahondaremos aquí en cuestiones metodológicas) tiene su potencial y su riesgo, elementos que muchas investigaciones de los últimos años vienen explorando cada vez con más detenimiento. De entre sus potencialidades, destacamos el hecho de que las publicaciones periódicas constituyen una herramienta clave para reconocer un contexto intelectual 
determinado. Las revistas permiten señalar quiénes eran los principales interlocutores en un determinado momento, y cuál era la posición de éstos, así como las diferencias que se suscitaban entre diversas figuras, e incluso son útiles para identificar los agrupamientos y las diferencias intelectuales que los atraviesan. Hay debates; hay críticas; hay citas; hay un ir y venir de nombres, en un diálogo que fluye y establece filiaciones y diferencias. Pero hay también un riesgo evidente: esos agrupamientos presentan diversas características. En algunas ocasiones, el que se expresa en la revista es un grupo, pero en muchas oportunidades se trata apenas de individuos con cierta proximidad teórica y/o ideológica. Del mismo modo, podemos encontrar allí a personajes del escenario intelectual, pero escasamente comprometidos con la línea general de la publicación. En cualquiera de esos casos, estamos obligados a cuidar las identificaciones simplificadoras. A veces el reconocimiento entre pares y el aprovechamiento del capital simbólico de un espacio o de un nombre es la principal causa de algunas extrañas - aunque frecuentes- combinaciones en sus índices. Por momentos, se incluyen artículos de autores con los que evidentemente no se comulga desde la línea editorial. Todo ello invita permanentemente a revisar el sentido de la revista.

Aún sabiendo estos riesgos, tomaremos a Valoraciones y la Revista de Filosofía como una unidad. En el caso de la primera, se trata explícitamente de la publicación de un grupo intelectual o cultural. No ocurre lo mismo con la Revista de Filosofía en la que, además, la gran cantidad de autores que pasaron por sus páginas hace más difícil la identificación. No obstante, en uno y otro caso, estamos convencidos de que, haciendo las salvedades del caso, en este tipo de publicaciones debe haber alguna comunión que convoque y reúna a los autores a participar del proyecto. En lo que sigue, nos guiamos por esta convicción, y hacemos las salvedades correspondientes, para comprender dos modos diversos de definir el saber filosófico que son también, a nuestro juicio, dos lenguajes diversos del reformismo universitario.

\section{VALORACIONES}

Si consideramos a sus cultores y autores más frecuentes, Valoraciones es una revista muy vinculada con el espacio universitario y, en ese marco, en especial con el espacio filosófico. Sin embargo, no se descuidan allí otras expresiones culturales como la literatura o las artes plásticas, que son una extensión de las opciones filosóficas por las que apuesta la revista. El tratamiento de la estética es moneda corriente en sus páginas, y la importancia otorgada al arte se justifica filosóficamente.

Valoraciones sale a la luz en La Plata, a mediados de 1923, por iniciativa de Héctor Ripa Alberdi, pero bajo la dirección de Carlos Amaya, y se edita hasta abril de 1928, con un total de doce números de aparición irregular, organizados en cuatro tomos. ${ }^{2} \mathrm{Al}$ año siguiente de iniciarse su publicación, Amaya renuncia a ese cargo y es reemplazado por Alejandro Korn, que ya entonces oficia como padre espiritual del grupo de intelectuales y artistas de La Plata y de la renovación cultural que buscan desplegar.

El nombre completo de la revista es Valoraciones. Humanidades, crítica y polémica, y lleva en la portada una leyenda que reza "Revista editada por el Grupo de Estudiantes 'Renovación”". 3 A pesar de manifestarse como una publicación de estudiantes, cuenta con una marcada presencia de autores que exceden esa categoría, empezando por Alejandro Korn. Y ello redunda también en otra característica, destacada por Rodríguez (1999): su marcado perfil academicista. Publican allí, además de los mencionados Amaya, Ripa Alberdi y Korn, Pedro Henríquez Ureña, Arturo Costa Álvarez, Francisco Romero y Carlos María Onetti, entre otros.

Cada número se divide en secciones: una, central, en donde predominan artículos referidos a cuestiones filosóficas y, en menor medida, estéticas; otra denominada "Bibliografía”, con novedades y reseñas, y una última que lleva por título "Comentarios", en la que se incluyen algunos temas de actualidad cultural. Los dos últimos números agregan una sección más, denominada "Celuloide", con breves artículos relativos a las artes plásticas.

Valoraciones se presenta como portadora de una tarea específica, desde su primer número y hasta el final: hacer efectiva, real, la Reforma Universitaria. Tal como afirman en la nota editorial del primer número, para 
sus editores, "la bandera de la Reforma [...], hoy no es más que el trapo descolorido que envilecen las traiciones de los mercaderes" ("La redacción”, Valoraciones,1923, p. 4). Ante ese símbolo gastado, la revista y el grupo que la impulsa encuentran su razón de ser.

La crítica no sólo alcanza una mirada desilusionada por el derrotero vivido (que no habría llevado sino a nuevas formas de lo mismo: a una Universidad en manos de niños o rutinarios profesores que ensayan una comedia, pasando por encima de algunas de las conquistas fundamentales, ${ }^{4}$ sobre cuya consideración y análisis se vuelve permanentemente en sus páginas): la crítica es también una mirada sobre el legado de la Reforma, sobre el intento de mantenerla aún como consigna, y allí es donde la revista busca inicialmente posicionarse, aunque con el tiempo y el suceder de sus números pueda plantearse, en sus páginas, la viabilidad de seguir apegada a la institución universitaria.

El primer número declara que "es necesario que los estudiantes terminen de una vez esos discursos apologéticos de las huelgas pasadas [...]. Ahora es menester despertarse porque ha llegado la hora del trabajo" (“La redacción", Valoraciones, 1923, p. 4).

La revista nace recogiendo el guante de la Reforma pero proponiendo con ello una definición particular: no parece importarle tanto si fue o no una gesta heroica, o si tuvo o no algo de lo que el relato más aceptado ha realzado, porque no es tiempo de apologías sino de trabajo. De ese trabajo dependen las posibilidades de posicionarse en el campo de las disputas académicas con una identidad bien definida.

La tarea a la que convoca Valoraciones gira en torno a la definición y a la afirmación de "Nuevos valores" (o, de otro modo, de "un espíritu nuevo"). En el primer editorial se habla de la "superación mental del estudiante y crítica del profesorado", ambas cosas -intuimos- íntimamente vinculadas. En esa renovación espiritual, entonces, radica la posta "reformista" a que apela Valoraciones. Y el vocabulario de 1918 se actualiza: "Este conglomerado heterogéneo de escuelas profesionales de índole meramente utilitario, fábrica de diplomas, sin unidad ni cohesión moral, no puede ser el alma mater de la nueva generación" ("La redacción”, Valoraciones, 1924, p. 315).

La relación de la revista con la Universidad es tensionante y en parte esa tensión se desprende de otra, la que se establece entre lo que es y lo que debe ser la Universidad. Y la revista se erige en juez y en guía: "En la actividad fundamental de nuestro país, las cuestiones universitarias asumen actualmente un papel preponderante” (“La redacción”, Valoraciones,1924, p. 315), decía el primer editorial. Pero antes

-acabamos de notarlo-, se recuerda permanentemente el déficit moral, y por qué no teórico, por el que atraviesa la Universidad actual: se llama a negar el profesorado, o a negar de su valor como alma mater. La Universidad aparece distante respecto del modelo a seguir. Hace falta otro motor; por eso, se señala a la juventud reunida en la revista y, al hacerlo, imperceptiblemente se evoca la filosofía, asignándole un perfil determinado. ${ }^{5}$

La definición de filosofía se elabora bajo la tensión entre el "positivismo" y el "antipositivismo". ${ }^{6}$ En el número dos, de enero de 1924, se recuerda y homenajea a Ripa Alberdi, recientemente fallecido, y se incluye el discurso que pronunciara en el Congreso Internacional de Estudiantes en México, el año anterior. Allí Ripa Alberdi es claro: "La juventud argentina marcha hacia la Universidad ideal por las rutas que le abriera la filosofía contemporánea" (Ripa Alberdi, 1924a , p. 111). Esa filosofía viene a liberar a la juventud "del peso de una generación positivista" (Íbid, p. 112). Había que beber de nuevas fuentes para encontrar las armas de la renovación que afecta, primero a la filosofía, después a la Universidad, y por último a la cultura toda. Entre esa renovación disciplinar y la superación de la "Universidad profesionalista" hay una continuidad estrecha que opera sobre el sentido mismo de aquel modelo "positivista”, y que construye su definición. En el discurso de la revista, el positivismo reinante en las últimas décadas es la causa del profesionalismo que condena a la Universidad, y por eso el reproche los aúna naturalmente. Desde esta perspectiva, son lo mismo, aunque el énfasis de las responsabilidades cambia de manera oscilante.

Junto con el pecado del profesionalismo se vislumbra otro, sin duda de mayor alcance: ese modelo de saber se ha puesto "al servicio de la muerte", como dice el químico Herrero Ducloux; ha educado el espíritu de los 
hombres para la muerte, en vez de educarlo para la vida, según señala Ripa Alberdi. Ante la guerra, el juicio se vuelve extremadamente lapidario y no reconoce distinción, ni precisión alguna.

Contra ese modelo se proclama "el despertar del espíritu" que trae la filosofía idealista. El clima de época, presente en la revista, sugiere un estado de crisis de valores, con el consecuente primado del escepticismo que se desprende del hecho de que hayan sido las pasiones y los intereses materiales los que guíen el esfuerzo humano. Se trata de un escenario propicio, en que cobra sentido una filosofía en manifiesta oposición al materialismo, a esta corriente que "dejó caer en el corazón argentino la gota amarga del escepticismo" ("La redacción”, Valoraciones, 1923, p. 5). Aunque política y económicamente el materialismo tenga diversos significados y derivas, la mirada que aquí se planta a nivel filosófico lo reconoce como único enemigo a combatir.

En ese marco entonces, y tal como reza el editorial del primer número, la conclusión de la Reforma depende de la renovación filosófica. Dos son las figuras que elaboran la estructura básica del planteo que la revista busca legitimar: Ripa Alberdi y Alejandro Korn. Si bien el primero fallece antes que el segundo número, el texto que mencionamos -el discurso preparado para el congreso de México- parece una muestra de los presupuestos básicos sobre los que se elabora la propuesta que seguirá la publicación.

Korn es, desde el principio, inspirador de la revista y de la juventud que la lleva adelante, aunque, luego del apartamiento de Amaya cobre mayor protagonismo, y claramente sea su pluma la responsable de las notas editoriales. $^{7}$

En los artículos publicados en la revista bajo su firma, Korn deja muy en claro que se propone ensayar un nuevo paradigma para la filosofía, que viene a reemplazar dos definiciones en disputa en el escenario contemporáneo. Para él, tanto el idealismo como el materialismo pecan de un reduccionismo con graves consecuencias. Y en ello, según puede observarse a partir de otros artículos, viene a plantear la posibilidad de retomar la tarea que el propio Kant dejara pendiente: "tras Kant [denuncia] aún persiste el malentendido" (Korn, 1924c, p. 9).

En ambos extremos, el monismo, en función del cual se considera ya el mundo de los objetos, ya el de los sujetos, termina por desconocer algún aspecto esencial. Se prefiere afirmar, por el contrario, que "sujeto y objeto no se conciben sino en relación mutua", y desde allí se postula un "dualismo trascendente" (Korn, 1924b, pp. 166-167). ${ }^{8}$ No obstante y en última instancia, la principal crítica recae sobre el monismo materialista, probablemente en parte porque éste ha tenido, en el momento, mayor acogida en la academia. ${ }^{9}$ En términos filosóficos, lo que Korn reclama, de cara al materialismo, es la dilución en la materia, de una condición indispensable y humanamente ineludible como la voluntad. Se niega a reducir la acción humana a la causalidad, y para hacerlo necesita sostener la voluntad como principio diferenciador. La actividad humana obedece a un motivo; es "expresión de la voluntad" (Korn, 1925-1926?, p. 105). Tal como lo había dicho a raíz del homenaje dedicado a Kant, para él es preciso volver a Kant; recoger esta doctrina "olvidada en los tiempos aciagos del positivismo y del materialismo económico, que enaltece la personalidad humana y la opone, como un centro de iniciativa libre, al juego de las fuerzas naturales” (Korn, 1926?a, p.11). ${ }^{10}$

Los niveles epistemológico, económico, e incluso metafísico se mezclan en estos debates. El materialismo económico, como lo llaman tanto Korn como en general el resto de los miembros de la revista, supone la disminución, o incluso el envilecimiento, de una voluntad humana que, esencialmente, escapa a las reglas de la naturaleza, como ajena a todo condicionamiento. La presencia de Kant atraviesa toda la revista y deja en claro, con su director, el objetivo de su propuesta filosófica: no volver a Kant; avanzar sobre lo que él había dejado pendiente, y asegurar lo que había quedado poco firme. No renegar de la ciencia, pero ajustar con la filosofía cuando de cuestiones humanas se trate. Para ello, echan manos a otros recursos: Ortega y Gasset, Bergson, Croce o Windelband figuran entre sus páginas, llamados a completar la obra iniciada por Kant. ${ }^{11}$

La exigencia es práctica y desde allí se buscan los recursos para convertirla en necesidad filosófica. Urge reconocer el "testimonio irrecusable de la conciencia", que nos da las pistas suficientes para afirmar la voluntad 
autónoma. Hay que resolver el problema del hombre, de la historia, de la cultura; hay que salir de la crisis que no conduce sino al nihilismo o al primado de una lógica utilitarista y mercantilista, y el único modo de hacerlo es reconstruyendo las bases filosóficas sobre las que estamos parados. Así lo dice Korn en "Nuevas bases". Según demanda la afirmación de este texto, si Alberdi escribió aquellas Bases en las que daba al futuro argentino un perfil economicista y utilitarista (como primeros pasos en el despliegue de una lógica positivista, en función, probablemente, de ciertas necesidades de su época), también con Alberdi, aunque ahora con el Alberdi de 1842, se puede atender a las necesidades del tiempo y del lugar en que se vive y se piensa. Y son precisamente el maestro platense y la revista que él preside quienes vienen a definir esas bases con claridad: "andamos en busca de un contenido ideal para nuestra vida [...]. En nuevas ideas se ha de expresar el sentido histórico de los nuevos tiempos" (Korn, 1926?b, p. 10). Una vez más, la historia queda en manos de la filosofía de la historia.

El problema de la hora es moral; por ello, es tarea de la filosofía. En ese sentido, en el mismo momento en que se define el presupuesto básico (el dualismo que no anula ni sujeto ni objeto, pero que fundamentalmente deja libre al sujeto), en ese momento -decimos-, se establece que la filosofía es la disciplina llamada a orientar el rumbo. La elevación de la filosofía, al lugar de saber superior y protagonista de la historia por venir, no puede darse sin antes definirla. Allí radica la disputa académica, que se hace muy evidente en el comentario de Korn a un trabajo de Alberto Palcos titulado La vida emotiva: "Altos son los fueros de la ciencia; la filosofía ocupa la misma jerarquía y no le está subordinada. El híbrido consorcio perjudica a una y a la otra. Frente al mundo objetivo está el subjetivo, frente a la energía física, la voluntad consciente, frente a la naturaleza, la cultura humana" (Korn, 1926?c, p. 286).

Cultura y naturaleza son conceptos no reñidos pero opuestos, que reclaman saberes diversos. La ciencia no puede, como pretenden muchos de sus contemporáneos, ocuparse de los valores. A su juicio, ésta sólo se ocupa del conocimiento mensurable y bajo ningún aspecto puede avanzar sobre el terreno de la axiología, sino a costa de la negación de la voluntad. El razonamiento no puede evitar el regreso al supuesto indiscutible que le sirve de punto de partida: lo humano es sinónimo de voluntad. La finalidad del hombre es la libertad, y he aquí el supuesto fundamental que debe regir toda reflexión sobre los asuntos humanos. A la ciencia como saber atenido a lo espacial-, se le escapa, en gesto de rebeldía, la personalidad humana.

En "El concepto de ciencia", Korn se expresa con precisión, e incluso elabora otro argumento para mantener distante la axiología respecto de la ciencia: ocuparse de cuestiones de valores no es postular verdades universales. Bien, justicia, belleza, todos los valores - dice- son subjetivos; significan algo diferente para cada uno de nosotros, y por ello no son generalizables. Mientras la ciencia busca acumular conocimiento, la axiología se renueva constantemente. ${ }^{12}$ En ese marco, la filosofía reviste una tarea especial y fundamental, aunque no por ello menos problemática y difícil de sostener sin nuevos supuestos: sistematiza todos los valores y busca la finalidad suprema a la que éstos se subordinan (esto es: la libertad). Para Korn, la filosofía llega incluso a ser una actitud.

En la misma línea se expresa Francisco Romero, quien a pesar de su escasa participación en la publicación, ${ }^{13}$ se ubica en la misma posición que su maestro y da cuenta de la problemática más general que inquieta a los intelectuales allí reunidos. Reseñando el texto de Carlos Astrada, "El problema epistemológico en la filosofía actual", Romero cuestiona la ausencia de toda consideración sobre los aportes de Rickert (quien, planteando las oposiciones naturaleza-espíritu, físico-psíquico, naturaleza-historia, filosofía naturalista-filosofía historicista, habría descubierto los "pares de conceptos que secretamente alimentan la dualidad del saber", combatiendo con ello "la concepción naturalista de la filosofía"; Romero, 1928, p. 238). En ese mismo sentido, un tiempo antes, Carlos Amaya había reseñado el libro de Rickert, Ciencia cultural y ciencia natural, afirmando acordar con el alemán en el modo de comprender ambos saberes: no muestran realidades diferentes, sino perspectivas distintas para el estudio de lo mismo, "dos principios formales en el proceder conceptual de las ciencias" (Amaya, 1924, p. 133). Algo muy parecido pronuncia uno de los últimos textos que publica la redacción: "La unidad de la ciencia es un dogma intangible del positivismo". Y a propósito de 
los "Premios nacionales a la ciencia y a las letras", insiste en incluir, como tercera categoría, la de "ciencias culturales" ("La redacción”, Valoraciones, 1928, p. 257).

Así, se conjuga una disciplina con un contenido específico, la filosofía, y una serie de conceptos que se van definiendo encadenadamente: libertad, vida, cultura. El fin de la vida, "impulso ingénito" -como lo llama Korn-, es la libertad, y éste se hace presente a través de la "cultura", expresión de la libertad creadora. La cultura es el modo en que lo más propio de la vida humana toma un lugar en la historia. La filosofía oficia allí como mediadora.

La juventud reunida en la revista reconoce que se tarea debe concentrarse en el plano filosófico-cultural, en el que cabe el trabajo "creativo" y, fundamentalmente, orientador de nuevos valores. La crisis es espiritual; la filosofía, definida como se ha hecho hasta aquí, se erige en el saber capaz de sacar a la humanidad de esa situación. De la mano de esta filosofía todo es novedad. Llamados a ser constructores de esta opción, los jóvenes próximos a estas definiciones se constituyen en una "nueva generación", que tiene en sus manos la tarea de diseñar una comprensión renovada del mundo y de lo humano. "Andamos en busca de un contenido ideal para nuestra vida", dicen, según ya recordamos.

En ese marco, el reformismo redefine sus contornos: sirve para nombrar a un grupo pequeño de jóvenes universitarios, aunque no necesariamente estudiantes, dedicados a la filosofía o a la "alta cultura", según las descripciones, y dispuestos a embanderarse con principios que se consideran académicamente renovadores. Las primeras notas de la revista se refieren a una "minoría selecta", llamada a influir en el destino del pueblo. Así lo plantea Carlos Amaya en el primer número, al reseñar el trabajo de Ortega y Gasset, España invertebrada, sentando algunas bases inconmovibles (Amaya, 1923, p. 44). ${ }^{14}$

La filosofía aparece acompañada del arte. La crisis de valores que hay que enfrentar se prolonga, a juicio de estos autores, también hacia el campo de los valores estéticos. En ese marco, el arte aporta en la búsqueda de la filosofía. Astrada se refiere, inspirándose en Ortega, a "la elaboración estética de nuestros contenidos espirituales" (Astrada, 1924, p. 185); Sánchez Reulet sostiene que "la realidad del artista es creada por su yo", por el trabajo interior de una consciencia en la que anidan los datos externos (Sánchez Reulet, 1925-1926?, p. 128).

En ese cuadro de la "alta cultura", a la que apela la revista a propósito de una definición de filosofía, se ensayan expresiones vanguardistas y latinoamericanistas para el arte. Sin adentrarnos en el asunto, advertimos simplemente que toda referencia a la renovación de los valores estéticos va acompañada de una reivindicación del arte autóctono, siendo varios los textos que se refieren a esta cuestión. ${ }^{15}$

A propósito de esto, y concluyendo el análisis de la publicación, cabe revisar el sentido mismo de aquel proyecto general en el que se inscribe la revista. Nos referimos al grupo Renovación y al hecho de que, tal como sus protagonistas los definen, su razón de ser tiene que ver precisamente con la elevación de la personalidad. Así, muchos de sus proyectos se articulan a la perfección con esta idea de la cultura y del saber filosófico. Retomando lo que plantea Graciano, lo que despierta la sensibilidad del grupo, y le sirve de incentivo, es la crisis que atraviesa la personalidad, mutilada por el capitalismo: "el aniquilamiento de la personalidad humana en una concepción utilitaria de la vida” (Graciano, 2017, p. 156). Hay que superar el empobrecimiento espiritual que condena la personalidad, bajo la afirmación de los valores de la burguesía. Para ello, la propuesta es, ciertamente, aristocratizante. Frente a la cosificación del sujeto y a la masificación, la cultura humanista, la filosofía de un grupo clarividente, el arte de los elegidos que pueden desplegar, en un lenguaje propio, los verdaderos valores de un "arte puro". Pensar la Reforma y concluirla, en ese discurso, es avanzar en el fortalecimiento de una elite intelectual que pone, en el centro de sus preocupaciones, la posibilidad de pensar al hombre con absoluta independencia respecto de sus necesidades e intereses materiales. La filosofía y el arte ofrecen el escenario propicio para esa reconstrucción. La Universidad también puede hacerlo si las condiciones cambian. Ese cambio es, precisamente, para ellos, la realización de los ideales de la Reforma. 


\section{Revista de Filosofía}

La Revista de Filosofía se publica en Buenos Aires entre 1915 y 1929, primero bajo la dirección de José Ingenieros, y luego con la colaboración de Aníbal Ponce que, en 1925, tras la muerte de Ingenieros, queda a cargo de esa tarea. Muchos elementos aproximan esta publicación a Valoraciones. Aunque de más extensa vida y producción, el rasgo "academicista" también la impregna desde el comienzo. Comparten muchos articulistas, aunque la frecuencia de éstos varía en proporción inversa: los más frecuentes en la Revista de Filosofía no lo son tanto en Valoraciones, y viceversa. Sin embargo, en lo que hace al objeto de este trabajo, notamos importantes diferencias.

Si bien esta revista comienza a publicarse, como dijimos, algunos años antes de los sucesos en Córdoba y sus repercusiones posteriores, 1918 es un año marcado por varias inflexiones. Quizás sea este acontecimiento, sin restarle importancia al efecto que sobre la revista tiene la Revolución bolchevique, el que marca un nuevo rumbo en el proyecto, que va desplazando el foco de atención hacia los sucesos políticos del momento. ${ }^{16}$ La Revista de Filosofía nunca renuncia a la elaboración y a la disputa en el plano teórico, aunque ésta se combina con la reflexión en torno a las implicancias políticas de las posiciones adoptadas en aquel nivel, y con una mirada más porosa, atenta a los sucesos de la hora. Y en esto la Reforma, decimos, funciona como bisagra. La revista se presenta en ocasiones como vocera de los acontecimientos sucedidos en Córdoba; se anima a relatarlos a través de las notas tanto de su redacción como de sus articulistas, y por supuesto, toma posición, elaborando una lectura que, a nuestro juicio, discrepa con la de Valoraciones. Dicha discrepancia, hipotetizamos yendo al nudo de nuestras preocupaciones, tiene que ver con un modo diverso de entender el conocimiento y la filosofía.

El número de julio de 1918 contiene los "Antecedentes sobre los sucesos de la Universidad de Córdoba”, un anti-relato frente a los "documentos oficiales y oficiosos", que provienen, principalmente, de La Nación. Tal como se describe en la revista, esos sucesos son resultado del enfrentamiento entre los elementos clericales, “agrupados en la sociedad semi-secreta 'Corda Frates' y los elementos liberales que desean renovarla de acuerdo con los resultados de la ciencia contemporánea” (Barreda Lynch, 1918, p. 135). En el relato de junio, habría sido esa misma institución católica la "que consiguió que los jesuitas y el clero presionaran a los electores por medio de sus madres, esposas e hijas...", para quedarse con el Rectorado. La Corda se constituye como protagonista y responsable de este capítulo, que pasa a ser uno más "del vasto plan católico para apoderarse de la educación argentina, desde la escuela primaria hasta la universidad" (Barreda Lynch, 1918, p. 136). En ese número se reproducen algunos documentos, discursos, entrevistas y declaraciones, entre ellas la famosa intervención de Nores, el Rector electo, en la que manifiesta que, de ser necesario, habría que masacrar a los estudiantes.

Del mismo modo, en los números sucesivos, la revista se muestra preocupada por los diversos acontecimientos del escenario universitario. En el número de enero de 1919, se incluyen los discursos se asunción de diferentes decanos de la UBA: Alejandro Korn en Filosofía y Letras, Estanislao Zeballos en Derecho y Ciencias Sociales, Eleodoro Lobos en Ciencias Económicas, y Julio Méndez en Ciencias Médicas; ${ }^{17}$ se reproduce la pastoral de Zenón Bustos, en la que el sacerdote cuestiona la manifestación en reclamo por la agresión sufrida por Barros, y denuncia la amenaza del maximalismo; se relata el conflicto judicial de Arturo Orgaz y Capdevila, en que se los separa de sus cargos de fiscales aduciendo ideas atentatorias contra el orden y se reproducen sus defensas; se incluyen conferencias vinculadas con el movimiento reformista y algunos manifiestos, así como la conferencia que Susini ofrece en La Plata, en marzo de 1921. En muchos casos los textos hablan por sí mismos; en otros, su inscripción en el contexto de publicación permite reconocer la posición de la revista. El más notorio en este último sentido es el caso de la pastoral, que va acompañada del trabajo de Ingenieros "Sobre la significación histórica del maximalismo", que no es sólo una explicación, sino también una defensa de ese movimiento. 
Esas narraciones (empezando precisamente por la primera, aquella en que Barreda Lynch se refiere a la Corda Frates) enfatizan tres elementos que recorren, a nuestro juicio, la lectura de la revista sobre la significación del acontecimiento universitario: su inscripción en una transformación educativa más general, la estrecha vinculación de ésta con la consideración de sus implicancias y exigencias sociales, y finalmente el antidogmatismo, como signo distintivo de toda su propuesta filosófica y educativa.

En lo que hace a la inscripción de la Reforma en el análisis más global de la educación argentina, la revista es contundente y sus definiciones se vinculan con una concepción del saber y del hombre. La reproducción del discurso ofrecido por Deodoro Roca ${ }^{18}$ en septiembre de 1920, en el marco de la Federación Universitaria de Córdoba, es muestra fiel de la complejidad de los postulados sobre los que se asienta la publicación. Una crítica a los científicos -que se apoya en las denuncias de George Nicolai, articulista frecuente de la revista- y al derrotero que, gracias a ellos, siguió la ciencia en el siglo XIX, son los motivos que elige el cordobés. Desde allí, advierte acerca de sus consecuencias en lo relativo a la organización de la educación y al perfil de la Universidad. También se piensan las posibilidades futuras del hombre. La ciencia se ha puesto al servicio de los intereses, diseñando un sistema educativo capaz de reproducir una división del trabajo que garantiza a unos pocos el dominio del mundo. "Desde entonces se distribuye con férrea consigna, por escuelas y universidades, un ejército resonante de asalariados intelectuales, de domésticos doctorados, de dómines verbalistas y pedantes, de parásitos de la cultura" (Roca, 1921, p. 236); ellos son los que se ocupan del saber en las instituciones, y éstas no tienen por fin más que la reproducción de ese sistema: "La tiranía de clase deviene un sistema cerrado, y la ignorancia es un resorte educacional, otro instrumento gubernativo" (Roca, 1921, p. 236).

Frente a ello, la Reforma es comprendida como respuesta al reconocimiento de una crisis que excede el ámbito universitario, y que puede sintonizar, sin inconvenientes, con la Revolución Rusa y sus transformaciones. Entre éstas, se destacan los aportes de Lunacharsky, de "la escuela unificada del trabajo", cuyo objetivo apunta a unificar al hombre, dividido por la ciencia del siglo XIX. Si en el texto de Roca la referencia al modelo ruso parece mediada por Taborda, D’Ors y María de Maetzu, la revista puede recoger esa piedra para lanzarla con mayor precisión. En el mismo número en que se publica el discurso de Roca, en octubre de 1920, se incluye el "Informe enviado al Comité Central Ejecutivo", por A. Lunatcharsky, y publicado en La Izvestia - por entonces el periódico que expresa la opinión del gobierno ruso-, al que se le antepone, en un claro gesto de apropiación, el trabajo de Ingenieros, "Las fuerzas morales de la revolución”. Allí, el director de la revista no sólo ensalza la Revolución, contra los múltiples ataques de sus enemigos, sino que además la resignifica como muestra contundente del triunfo de "nuevas fuerzas morales". Algunos números antes, en julio de 1920, también publica algo al respecto, en su artículo "La reforma educacional en Rusia”, acompañado por una serie de documentos que completan la información sobre ese modelo educativo. La experiencia soviética es interpretada como un escalón más del movimiento progresivo de la historia, comenzado en el Renacimiento, que por fin viene a completar la obra que ni el mismo siglo XIX, con el persistente predominio de la "injusticia de clase", había logrado. Se trata de la "educación integral" que, al tiempo que busca conciliar lo humano, en la no distinción radical entre trabajo manual e intelectual, quizás por ello mismo, tiene muy presente que la educación debe estar íntima, pero progresivamente, vinculada con el trabajo. De allí se desprende también una idea de la educación superior que enfatiza la autonomía de su gobierno y, junto con ello, se elogian experiencias relativas a la educación popular y la extensión universitaria. Tal como se presenta allí, en aquel modelo, el sentido último de la formación universitaria está orientado a crear los instrumentos e instituciones destinados a formar culturalmente al proletariado. En los documentos que acompañan el texto de Ingenieros, Lunatcharsky plantea claramente las funciones del proletatkultur: "su objeto no es atraer al obrero hacia la universidad, sino llevar la educación universitaria al centro obrero, al sindicato [...]. La Nueva Rusia ha comprendido que las revoluciones sólo son duraderas cuando actúan sobre la conciencia del pueblo (Ingenieros, 1920, pp. 85-86). Sin apoyarse completamente en el pedagogo ruso, en otros artículos Ingenieros sigue desarrollando las mismas nociones sobre la educación, agregando 
o precisando algunos elementos. El número de mayo de 1921 incluye un artículo de su autoría, titulado "Nuevos ideales de la educación", que reúne algunas ideas elaboradas con el objetivo de presentarlas en el Congreso Nacional de Maestros. En ese trabajo, vuelve a enfatizar el vínculo de la educación con la justicia y con la "utilidad social"; vuelve a cuestionar las condiciones artificiales que devienen de los privilegios, y a reclamar en favor de las inclinaciones naturales, confiando, sin embargo, en el poder de la selección natural (Ingenieros, 1921a, p. 455). La naturalidad en el desarrollo de las capacidades se da la mano con la utilidad y el bien social, combatiendo no sólo la artificialidad de la división del trabajo, sino también el forzamiento en la educación.

Otros autores, como Ernesto Nelson y Ramón Melgar, son importantes exponentes de la revista en lo que hace a cuestiones de educación general. Matizando el tono social y político tan explícitamente afín al modelo educativo ruso, se expresan en favor de una reforma de todo el sistema educativo, a fin de evitar sus nocivos efectos en contra de la justicia social. En el planteo de Melgar, resulta interesante notar cómo avanza sobre la cuestión de la ciencia y sus efectos. Su reclamo por una educación moral, como base de la reconstrucción de la humanidad en crisis (o como condición de la orientación del pueblo hacia el "porvenir"), se asienta en un esquema que ubica a la ciencia como condición de esas definiciones. Melgar insiste en que, si los desarrollos científicos condujeron a la guerra, la causa radicó en una mala interpretación de los mismos. Y, consecuentemente, puede apostar por una "filosofía científica", siguiendo el modelo de Ingenieros, que es condición de toda comprensión de la moral. Esa búsqueda no implicaría, en absoluto, el retiro hacia un modelo de racionalidad o saber reñido con la ciencia. La moral -dice- es un hecho puramente humano y ello lo ha probado, precisamente, la filosofía científica (Melgar, Revista de Filosofía, 1920, p. 404).

Esa inspiración en el modelo ruso, en base a la cual el conocimiento se vincula con las necesidades sociales, es en estos autores fundamento de la consideración no solo de la universidad y de la educación en general, sino también de la filosofía misma. La noción de "filosofía científica", que inicialmente surge de las investigaciones en torno a la psicología, ${ }^{19}$ se precisa en el marco de las preocupaciones referidas a la educación en el nuevo contexto, estrechándose el vínculo entre la filosofía y la experiencia social. Y desde ese punto de vista, el juicio contra la filosofía especulativa es lapidario. Pero vayamos lentamente.

Son numerosos los trabajos en los que se insiste en este modo de comprender la filosofía. También Ingenieros estrecha la relación entre ese saber y la experiencia, tal como lo proclama el editorial con que se abre la revista en 1915. Esa misma idea es expresada también por un cordobés ligado a la Reforma, en los días en que comienza el conflicto:

Como la filosofía no es más que la carrera de la inteligencia cabalgando sobre la experiencia, al través de la realidad, es inevitable la conclusión de que mientras no aceleremos el ritmo de la vida social por la mayor densidad del grupo, el pensamiento argentino no sólo no habrá salido del balbuceo filosófico, sino que caerá en la falacia de imaginar que la filosofía se logra en la agradable divagación de admoniciones generosas o de exhortaciones trascendentales (Orgaz, 1918, p. 117).

En ese y otros trabajos, Orgaz reniega de toda posible definición especulativa de la moral. Muchos de sus trabajos resumen bastante bien la posición de la revista al respecto: en tanto que se pretende ciencia puramente normativa, la moral resulta falsa o ilusoria, tendiente a legitimar las costumbres de los grupos dominantes.

Esa noción de la ciencia y de filosofía es, a nuestro juicio, uno de los principales elementos de disputa con otras posiciones "reformistas". Nuevamente, Orgaz afirma que el modo de evitar que la Reforma se convierta en un "nuevo rodaje de la máquina oficinesca" es solidarizando la filosofía con la ciencia, la metafísica con la experiencia, "para que las hipótesis florezcan en el suelo de la realidad” (Orgaz, 1922, p. 434). ${ }^{20}$ Retomando el esquema de Ingenieros sobre la organización de la Universidad, la filosofía que debe articular los otros saberes universitarios, y por ello su redefinición, es fundamental en esta nueva Universidad a la que se aspira. La Universidad tiene que volver a ser pensada a partir de la gran transformación de valores que conmociona al mundo y los saberes, atendiendo a las novedades recientes. La filosofía, un saber reflexivo sobre 
el conocimiento y su destino, y sobre los valores en sus múltiples manifestaciones, viene a constituirse en guía en este sentido:

Social por su origen [decía Orgaz], la filosofía lo es también y en grado eminente por su función [...]. Si la filosofía es la cristalización trascendente de un cierto concepto social acerca del universo y de la vida, sirve a la vez de instrumento para la lucha de los intereses y de los ideales en el seno de cada civilización (Orgaz, 1922, p. 434).

Y convoca, de este modo, a reconocer que la cuestión social era asunto de la filosofía, en tanto cuestión moral. ${ }^{21}$ Con esa base Orgaz, como tantos otros en la revista, aprovecha para rechazar toda expresión "antiintelectualista" de la filosofía "irracionalista", que la vuelve "dogmática y arbitraria" (Orgaz, 1922, p. 449). Contra ellas, reclama una nueva "cultura" para la Universidad, en donde las ciencias y la filosofía deben solidarizarse y articular un nuevo modelo. La filosofía debe darse la mano con la ciencia y, a través de ella, con la experiencia. Se trata, para el cordobés, de una filosofía que indefectiblemente termina comprometida con la transformación social. De esa realidad extrae sus insumos, y en función de ésta debe proclamar sus verdades. En lo que hace a la Universidad, se trata de una institución en donde la filosofía pasa a ocupar un lugar fundamental. ${ }^{22}$ Esa noción de la filosofía, a partir de la cual se propone una determinada organización de los saberes y las universidades, reaparece en varios trabajos, y puede ser considerada sin mayores problemas como un posicionamiento de la revista. De hecho, la propia publicación se organiza en base a ese sentido, desde su nombre, Revista de Filosofía. Cultura, ciencia, educación (en que la filosofía aparece como el gran paraguas sintetizador), hasta el contenido de sus trabajos y la organización de su índice. En éste puede verse cómo conviven armónicamente artículos referidos a las ciencias naturales y sociales, con otros en los que se atiende a debates y posicionamientos filosóficos, aunque también culturales y educativos.

Por otra parte, la crítica al idealismo es explícita, y ello puede ser leída como expresión de sus diferencias con el tono que va tomando el reformismo en otros grupos y orientaciones. Además de Ingenieros y Orgaz, otros autores frecuentes se pronuncian al respecto, sobre todo cuando, pasados unos años respecto de los acontecimientos iniciados en Córdoba, se ven ya algunas consecuencias que contradicen en mucho lo que, para ellos, eran los objetivos de la Reforma. Para José Katz, la ignorancia acerca de los problemas sociales está a la base de la construcción de "una filosofía ramplona y transhumante, mezcla de ingenua unilateralidad de especialistas y de pavorosa ineptitud para alentar ideales de ennoblecimiento colectivo" (Katz, 1929, p. 298). Narciso Laclau, atendiendo a otro aspecto, denuncia los intereses que, calladamente, sustentan las posiciones idealistas, y afirma que "fuerza es confesarlo: el idealismo argentino solo puede existir en la región amable de la teoría” (Laclau, 1924, p. 41). Aníbal Ponce también dejaba en claro sus diferencias en 1928: "Las enseñanzas del 'novecentismo', la 'nueva sensibilidad', la 'ruptura de las generaciones', no eran más que vaguedades que lo mismo podían servir - como quedó demostrado- a un liberalismo discreto que a una derecha complaciente" (Ponce, 1928, p. 290). ${ }^{23}$

Para ellos, que la ciencia fuese el modelo implicaba conjurar el dogmatismo. "El espíritu científico [decía Ingenieros] excluye todo principio de la autoridad [...]. Enseñar una ciencia no es transmitir un catálogo de fórmulas definitivas, sino desenvolver la aptitud para el perfeccionamiento" (Ingenieros, 1922, pp. 304-305). Conscientes de la crítica que llega desde posiciones enfrentadas, por la supuesta pretensión de generalidad y verdad de la ciencia, dan vuelta el argumento. Al rechazar la experiencia, mirado desde esta óptica, el idealismo se queda sin posibilidad de novedad. Aplicada al espacio de las experiencias sociales, la permanente modificación de la realidad social se convierte en el motor de renovación de los ideales. Mantenerse ignorantes de aquellos cambios, implica atarse a "viejos ideales", pues “cada ideal sólo será legítimo donde sean efectivas las verdades que lo engendran” (Ingenieros, 1922, p. 306). ${ }^{24}$

De este modo, las páginas de la revista están atravesadas por una síntesis entre ciertas consideraciones sobre la ciencia (en donde vuelven a recordarse las condiciones epistemológicas de todo saber), y una propuesta de tareas propias de la Universidad. Y con esto se denuncia algo pendiente desde 1918, tal como lo recuerdan las palabras de Roca. En esta revista aparece también -y de manera contundente- la crítica a 
la profesionalización, pero lo que se ve allí es el descuido que ella supone respecto de la ciencia, definida a partir de los principios que fuimos desplegando aquí. Así por ejemplo, Leopoldo Maupas lo dice en 1921: la crisis universitaria aún no está resuelta porque la Universidad no prepara para la ciencia y porque no se hace de la carrera de investigación una opción redituable. Junto con la defensa de un modelo científico de cierta rigurosidad, vuelve a aparecer la reflexión sobre la justicia social. El reclamo contra la profesionalización, si supone un respeto a las condiciones estipuladas para el quehacer científico (y una defensa de éste y de sus reglas), no se olvida en absoluto de la consideración de sus efectos sociales.

Conviven aquí una defensa de la ciencia como garantía contra los devaneos del idealismo, con la impronta de una lectura materialista del derrotero de la Universidad, que advierte las implicancias sociales del modelo de saber que allí se imparte. Dice Ingenieros:

Patrimonio común de la sociedad, las ciencias no deben constituir un privilegio de castas herméticas ni es lícito que algunos hombres monopolicen sus resultados en perjuicio de los demás. El único límite de su difusión debe ser la capacidad para comprenderlas; el único destino de sus aplicaciones, aumentar la común felicidad de los hombres y permitirles una vida más digna (Ingenieros, 1922, p. 303).

Y esto se vincula, sin duda, con las reflexiones del autor sobre las relaciones entre educación y trabajo, elaboradas a la luz de las experiencias soviéticas.

Son varios los artículos que se detienen en este punto y que plantean, manteniendo la distinción entre "universitarios" y "obreros", la necesaria complementación entre ambos sectores. Ese, incluso, es un costado significativo en los relatos de 1918. Con esto se configura también una imagen de la juventud como sujeto parado en la frontera que divide dos mundos casi inconmensurables todavía: puede asomarse y proponer objetivos conjuntos, haciendo de las masas, su vida y sus valores, un objeto de exploración y un objetivo de sus saberes.

Si bien en términos teóricos no parece viable destacar un grupo selecto ocupado del saber, que concurre a las universidades (y con ello, se insiste en el vínculo entre saber y utilidad social), la mirada reticente a los fenómenos políticos y sociales nacionales, y en particular a la democracia, parece sugerirle, a nuestros autores, la necesidad de ser cautos en la ampliación de las fronteras académicas. Es probable que la extensión universitaria y la universidad popular, ideas caras al reformismo y que están presentes en varios de los artículos de la Revista de Filosofía mucho más que en la otra publicación, deban ser comprendidos en este marco. Si bien los saberes se definen en función de su vinculación con la historia y las necesidades sociales, y pueden ser llevados a los sitios en los que éstos revistan alguna utilidad, se desarrollan en ámbitos en los que, si ya no prima, a su juicio, una división arbitraria de capacidades, su acceso aún depende de cierta "selección". No es la clase; no es una capacidad innata e individual lo que distingue; es la inclinación para la ciencia y el respeto de las reglas institucionales que impone ese saber. De lo contrario, ya lo saben, la universidad podría transformarse en "fábrica de títulos".

Si hay mucho acuerdo en torno al método y al paradigma que rige el saber que debe impartirse en la Universidad, conviven allí diversas posiciones políticas, como la exaltación del esquema ruso, acorde a una mirada complaciente de las transformaciones soviéticas y hasta del accionar revolucionario, con otras posiciones más próximas a resaltar el vínculo entre los saberes científicos y su utilidad en términos de reformas sociales tendientes a una transformación progresiva y pacífica. Los años inmediatamente posteriores a 1918, aquí privilegiados, presentan una fuerte inclinación de tono combativo. Con el paso del tiempo, con las transformaciones políticas, con el fallecimiento del director, con la creación de la Unión Latinoamericana (ULA), las preocupaciones y perspectivas van mutando. Ya lo advertimos, el tamaño de esta empresa editorial, la cantidad de números editados y la cantidad de años que se mantuvo viva, vuelven difícil hacer una única descripción que lo englobe todo. Pero en ese marco, es interesante observar un fenómeno que vemos aparecer en los últimos números de la revista, y que convoca nuevamente el tema de la Reforma: pareciera que entonces, ya próxima a desaparecer, y ante nuevas condiciones políticas, la revista busca ajustar su posición. En el número de mayo de 1928 se incluye un artículo del comunista Paulino González Alberdi, en el 
que cuestiona duramente el movimiento reformista. Como representante de esa expresión, lee la Reforma como manifestación de la pequeña burguesía que intenta desplazar a las familias patricias, de los puestos universitarios. La constitución de un "proletariado intelectual” está en sintonía con el poder que va ganando el radicalismo y no sirve sino para denunciar la distancia entre este sujeto político y el proletariado. La crítica de González Alberdi no sólo alcanza al movimiento reformista de 1918, sino que además se expande sobre sus repercusiones.

Y si, ya para esta fecha, el reformismo se ha trocado en latinoamericanismo, la caracterización del comunista también alcanza estas expresiones. El escenario entonces es muy diverso respecto del de 1918, y esa traducción a latinoamericanismo trae consigo la necesidad de nuevas apuestas o tomas de posición. La revista, que cuenta con figuras destacadas del espacio intelectual, es muy consciente de ello. Atentos al contexto de publicación encontramos que, en el número de septiembre-noviembre de 1928, se incluye un texto de Raúl Haya de la Torre, donde el peruano responde las críticas del comunista, identificándolo como un intelectual preocupado "por ceñirse rígidamente a una ortodoxia". En el debate que recién comienza, la Reforma Universitaria tiene su lugar. Haya de la Torre defiende la necesidad de reparar en los hechos, en las amenazas que sufre la región, presa del imperialismo que se desprende de un capitalismo ajeno. El liberalismo se ha retrasado en América; las revoluciones han quedado inconclusas, y esa experiencia permite pensar que la Reforma Universitaria se constituye, por fin, en el advenimiento del liberalismo: "es la efectiva revancha del auténtico liberalismo intelectual exigido para el desarrollo de nuestros pueblos” (Haya de la Torre, 1928, p. 127). Todo lo que hay de pequeñoburgués en el movimiento reformista toma, a juicio del peruano, un signo positivo. Las particularidades del escenario señalan allí, en la gesta de un sector reducido y burgués de la sociedad, la condición de posibilidad de que los pueblos latinoamericanos tomen consciencia del peligro de imperialismo. La Reforma, producto de las clases medias, es un aporte fundamental a la causa del antiimperialismo y, por ello, "es uno de los movimientos americanos más trascendentales" (Haya de la Torre, 1928, p. 133).

En esa disputa, la revista parece asumir una posición en favor de Haya de la Torre. Aunque ello no es explícito, su proximidad con la ULA permite pensar de este modo. Pero incluso, va más allá. En ese mismo número en que se publica el artículo de González Alberdi, encontramos un valioso trabajo de Ponce, "Un examen de conciencia”. Allí la Reforma aparece como la coronación de un proceso comenzado con las guerras de la independencia colonial. Haciendo pié en un relato de base materialista, Ponce no repara, sin embargo, en los intereses de clases que podrían haberse reconocido como factores determinantes de las diversas luchas. Y eso vale para la "nueva generación latinoamericana". Destaca en ella la capacidad de descubrir, en la Revolución Rusa, un ideal que le permite elevarse. Sin ser parte del proletariado, dice, se percató de la crisis por la que atravesaba su tiempo, pero precisamente por no ser parte del proletariado, también pudo dejarse seducir por los grupos conservadores. La Revolución Rusa planteó una oposición de la que ya no pueden liberarse: o burguesía o proletariado. Y la Reforma queda atrapada allí entre los dos extremos: una reforma pedagógica, funcional a la burguesía, y otra verdaderamente revolucionaria, "que echa abajo las columnas de la sociedad en que vivimos" (Ponce, 1928, p. 290). Aun reconociendo ese origen que la liga íntimamente al liberalismo, la Reforma está llamada, para él, a avanzar sobre esos pilares.

\section{Disputas}

El recorrido por las dos publicaciones parece mostrar dos opciones dentro del reformismo que, como anticipábamos, se construyen a partir de dos definiciones de la filosofía, en el marco general de los saberes universitarios. Si de un lado se comprende el rol central que la hora le asigna a la filosofía, como constructora de valores, en lo que se evalúa como momento crítico por el empobrecimiento sufrido en este nivel, en el otro, la centralidad de la filosofía va de la mano de una definición según la cual la base de esa condición está constituida precisamente por los aportes teórico-metodológicos de las ciencias naturales. En un caso, el protagonismo de la filosofía se desprende de una valoración moral, hecha por los mismos filósofos; en el otro, 
se deriva de algunos elementos en los que confluyen otros saberes, insumos de la filosofía y, explícitamente, consideraciones ante las coyunturas políticas. En un caso, el reformismo es un movimiento cultural, expresión de una elite, que se retroalimenta permanentemente en la medida en que se reconoce aislado de otras manifestaciones sociales. En el otro, si hay una minoría selecta, se advierte la implicancia política de las verdades a las que arriba y su utilidad social.

Se trata entonces de dos matrices filosóficas completamente distintas que, no obstante, conviven en un mismo escenario intelectual y comparten muchas de sus preocupaciones (entre ellas, una muy relevante es la de la Universidad, incluida la lectura y conclusión de la Reforma). Pero los modos en que se definen esas inquietudes son diversos, y las respuestas también.

En el caso de Valoraciones, la condena de la Universidad atraviesa toda la publicación, advirtiendo tanto las reformas regresivas de los estatutos como las prácticas anquilosadas. Representación en los órganos de gobierno, sistemas de elección de representantes, exámenes, clases... todo es expresión de que la Reforma no ha ocurrido aún y pone en tensión su viabilidad desde las aulas mismas de la Universidad. Difícilmente la Universidad de entonces (con muchos signos, para ellos, de contra-reforma) puede marchar hacia donde debe ir, marcando el rumbo de la historia. Con relativa autonomía, la revista sí se propone esa tarea. ${ }^{25}$ En efecto, es probable que Valoraciones sea más dura en su crítica a la Universidad de entonces que la Revista de Filosofía. La formación del grupo Renovación es, de hecho, una respuesta ante ese malestar. Pero ello no se agota en respuestas ante la contrarreforma. En Valoraciones conviven y son identificados los males de la Universidad: el conservadurismo y el profesionalismo/positivismo. La propuesta, entonces, va de la mano de una nueva Universidad que revise esos presupuestos filosóficos. Tal como la presentan, la filosofía que proponen es algo tan diverso a todo lo que ocurre en esa Universidad cientificista que allí no hay maestros ni guías. Entonces, la filosofía aparece como propuesta de reclusión, pensamiento cultivado entre pocos, para luego orientar la moral de muchos, aunque de ese último paso aún casi no se hable. Pareciera que la revista se contenta con dirigirse a los protagonistas de esta tarea: los filósofos y artistas próximos, hacedores de la nueva cultura.

La Revista de Filosofía, en cambio, pareciera querer potenciar su carácter universitario. Una muestra de ello es la referencia de cada articulista como profesor de alguna universidad o instituto educativo. Son parte de esa Universidad que se cuestiona, sin ensayar opciones por fuera de ésta (o mejor, reconocen explícitamente que tienen una función allí). La propuesta de reestructuración de los saberes y facultades es muestra de ello. La filosofía que proponen, bajo las condiciones que proponen, no sobrevive sino difuminándose por toda la Universidad, y llegando, quizás transfigurada, a los más diversos ambientes. Sin ese diálogo, este saber pierde también su objeto. En la revista, en ese sentido, hay alternativas, como la extensión o la Universidad popular; pero éstas se ensayan para un público diverso al de la academia.

Tal como lo plantea Valoraciones, dijimos, concluir la Reforma supone construir e instituir un nuevo sentido de la filosofía, y darle a ésta un valor protagónico en la Universidad y en la cultura. Terminar la Reforma es combatir un modelo que condena la cultura. Concluir la Reforma es reconocer la centralidad de un tipo de saber atento a la crisis cultural, pero ajeno completamente a sus causas o implicancias sociales. Es instaurar, por fin, el reino de un saber abstracto pero con capacidad directiva. La revisión de la Revista de Filosofía permite matizar la descripción negativa sobre la que se asienta Valoraciones. No toda opción afín a la ciencia es partidaria del cientificismo, ni mucho menos de la profesionalización, aunque las razones de la condena sean diferentes. Concluir la Reforma es, para la Revista de Filosofía, reconocer la implicancia social del conocimiento, su íntima vinculación con las condiciones y transformaciones materiales. Así, de una vez, de lo que se trata es de formas diversas de entender el conocimiento (y en ese marco, de entender las jerarquías académicas, aunque también sociales y políticas). Concluir la Reforma es disputar su sentido. En ese contexto, triunfar es instaurar un sentido para la filosofía. 


\section{FUENTES PRIMARIAS}

Amaya, C. (1923). “España invertebrada de José Ortega y Gasset”. En Valoraciones, tomo I (año 1, nº 1), pp. 43-47.

Amaya, C. (1924). "Ciencia cultural y ciencia natural de Enrique Rickert”. En Valoraciones, tomo I (año 1, nº 2), pp. 129-133.

Astrada, C. (1924). "El nuevo esteticismo”. En Valoraciones, tomo I (año 1, nº 3), pp. 178-185.

Barreda Lynch, J. (1918). "La Corda Frates en la Universidad de Córdoba. En Revista de Filosofía (año 4, 4-6), pp. 135-160.

Bermann, G. (1924). "La España invertebrada”. En Revista de Filosofía (año 10, 1-3), pp. 161-174.

Haya de la Torre, V. (1928). “En el XI aniversario de la Reforma”. En Revista de Filosofía (año 14, 4-6), pp. 131-133.

Ingenieros, J. (1915). "Para una filosofía argentina”. En Revista de Filosofía (año 1, 1-3), pp. 1-6.

Ingenieros, J. (1920). “La reforma educacional en Rusia”. En Revista de Filosofía (año 7, 4-6), pp. 70-143.

Ingenieros, J. (1921a). "Verdad-ciencia-ideal”. En Revista de Filosofía (año 8, 1-3), pp. 299-307.

Ingenieros, J. (1921b). “Las fuerzas morales de la revolución”. En Revista de Filosofía (año 8, 1-3), pp. 273-287.

Ingenieros, J. (1922). “Nuevos ideales de la educación”. En Revista de Filosofía (año 8, 1-3), pp. 454-461.

Katz, J. (1929). "El devenir de la reforma universitaria”. En Revista de Filosofia (año15), pp. 293-307.

Korn, A. (1924b). “Esquema gnoseológico”. En Valoraciones, tomo I (n³), pp. 164-168.

Korn, A. (1925-1826?). “El concepto de ciencia”. En Valoraciones, tomo II ( $\left.{ }^{\circ} 8\right)$, pp. 100-114.

Korn, A. (1926?a). “Kant”. En Valoraciones, tomo II (nº 6), pp. 5-13.

Korn, A. (1926?b). “Nuevas bases”. En Valoraciones, tomo II (n 7), pp. 3-11.

Korn, A. (1926?c). “La vida emotiva, por Alberto Palcos”. En Valoraciones, tomo II (n 9), pp. 282-286.

Korn, G. (1925-1926?). “Hacia un arte americano”. En Valoraciones, tomo II (nº 8), pp. 66-69.

La redacción (1924a). "La contrarreforma”. En Valoraciones, tomo I(n³), pp. 314-315.

La redacción (1923). "Intenciones". En Valoraciones, tomo I (n¹), pp. 3-5.

La redacción (1927). “Primer Salón de Escritores”. En Valoraciones, tomo II(n 10), pp. 73-74.

La redacción (1928). “Los premios nacionales”. En Valoraciones, tomo II (n 12), pp. 255-257.

Laclau, N. (1924). “La enseñanza y la investigación”. En Revista de Filosofía (año 10, 4-6), pp.38-41.

Melgar, R. (1920a). “La reeducación de la humanidad”. En Revista de Filosofía (año 6, 1-3), pp. 383-408.

Melgar, R (1920b). "La educación moral”. En Revista de Filosofía (año 6, 4-6), pp. 306-399.

Nelson, E. (1921). “La cuestión social y la cultura”. En Revista de Filosofía (año 7, 1-3), pp. 394-425.

Nicolai, G. (1925). “El sentido filosófico de la teoría de la relatividad”. En Revista de Filosofía (año 9, 4-6), pp. 2-26.

Orgaz, R. (1915). “Moral y sociología”. En Revista de Filosofía (año 1, 1-3), pp. 403-415.

Orgaz, R. (1918). "Filosofía y filodoxia”. En Revista de Filosofia (año 4, 1-3), pp. 116-119.

Orgaz, R. (1922). "La función sintética de la universidad”. En Revista de Filosofía (año 8, 1-3), pp. 432-451.

Ponce, A. (1928). "Un examen de conciencia”. En Revista de Filosofía (año 14, 1-3), pp. 279-292.

Revista de Filosofia. Cultura, ciencia, educación (1915-1929), Buenos Aires.

Ripa Alberdi, H. (1924). “Por la unión moral de América”. En Valoraciones, tomo I (n²), pp.110-115.

Roca, D. (1921). “La universidad y el espíritu libre”. En Revista de Filosofía (año 7, 1-3), pp. 234-239.

Romero, F. (1928). “El problema epistemológico en la filosofía actual de Carlos Astrada”. En Valoraciones, tomo II(nº 12), pp. 235-244.

Sánchez Reulet, A. (1925-1926). “Poiesis”. En Valoraciones, tomo II (nº 8), pp. 128-129.

Susini, T. (1921). “Evolución universitaria y social”. En Revista de Filosofía (año 7, 1-3), pp. 205-228.

Taborga, B. (1924). “Filosofía del diletantismo”. En Valoraciones, tomo I (año 1, n³), pp. 197-203. 
Valoraciones. Humanidades, crítica y polémica (1923-1926), La Plata.

\section{FuENTES SECUNDARIAS}

Artundo, P. (2010). "Reflexiones en torno a un nuevo objeto de estudio: las revistas". En IX Congreso Argentino de Hispanistas, Disponible en http://www.memoria.fahce.unlp.edu.ar/trab_eventos/ev.1028/ev.1028.pdf

Bustelo, N. (2013). "La juventud universitaria de Buenos Aires y su vínculo con las izquierdas en los inicios de la Reforma Universitaria (1914-1922)”. En Izquierdas (n 16), pp. 1-30.

Delgado, V. (2014). “Algunas cuestiones críticas y metodológicas enhttp://w relación al estudio de revistas”. En: Delgado, V., Mailhe A. y Rogers, G. Tramas impresas. Publicaciones periódicas impresas, La Plata, FAHCE/ UNLP. Disponible en ww.libros.fahce.unlp.edu.ar/index.php/libros/catalog/book/33

Graciano, O. (2014). “Alejandro Korn y las humanidades en la Universidad Nacional de La Plata”. En Archivos de Ciencias de la Educación (año 8, $n^{\circ}$ 8, $4^{\circ}$ época), pp. 1-16.

Graciano, O. (2017). "La filosofía en la ciudad: Alejandro Korn y las experiencias culturales del Grupo Renovación en una capital de provincia”. En Izquierdas (n³4), pp. 150-178.

Rodríguez, F. (1999). "Inicial, Sagitario y Valoraciones. Una aproximación a las leras y la política de la Nueva Generación Americana”. En Sosnowski, Raúl (ed.). La cultura de un siglo. América Latina y sus revistas, Buenos Aires, Alianza, pp. 217-248.

Rossi, L. (1999). “Introducción”. En Revista de Filosofía, Bernal, UNQ.

Ruvituso, C. (2013). "Pensamiento filosófico, inserción universitaria e idearios políticos en Alejandro Korn y Coriolano Alberini”. En Frederic, S., Graciano, O., Soprano, G. (coords.). El Estado argentino y las profesiones liberales, académicas y armadas, Rosario, Prohistoria, pp. 113-140.

Ruvituso, C. (2015). Diálogos existenciales. La filosofia alemana en la Argentina peronista (1946-1955), Madrid, Iberoamericana.

\section{Notas}

1 Doctora en Ciencias Sociales y Humanas por la UNQ, Profesora asistente en la cátedra de "Filosofía Argentina y Latinoamericana” de la Facultad de Filosofía y Humanidades de la UNC, e Investigadora Asistente en CONICET. Email: carlagalfione@yahoo.com.ar

2 Cabe aclarar que no es fácil fechar los diversos números, dado que esa información no está disponible en todas las portadas. En la medida de lo posible, consignamos esos datos, pero no siempre son fehaciente, porque se derivan de una triangulación de diferentes elementos (por ende, cuando la fecha no es segura, anotamos el año sugerido seguido de signo de pregunta).

3 "Renovación" es el nombre del grupo reunido gracias al impulso de Ripa Alberdi, y que lleva adelante una serie de actividades culturales en La Plata, destinadas a la difusión de la cultura. El grupo toma cuerpo con la creación, en 1921, de la Compañía Teatral Estudiantil Renovación, a la que luego se le suman otros proyectos como la revista Valoraciones y la editorial Renovación. Sobre este tema, ver el detallado estudio de Graciano (2017).

$4 \mathrm{Al}$ respecto, en el número de abril de 1924, por ejemplo, un artículo de la redacción, titulado "La contra-reforma”, relata una iniciativa de delegados de la Facultad de Derecho, en el Consejo Superior de la UNLP, en favor de limitar la participación estudiantil en la elección de autoridades académicas.

5 Advirtamos que, al referirse a la Universidad, los articulistas abandonan la generalidad y hablan de la Universidad de La Plata en la que, ya desde 1920, se producen importantes conflictos entre sus autoridades y los embanderados del Reformismo.

6 Resalto las comillas porque fue así como los protagonistas de entonces lo calificaron, y considero necesario, como estudiosa de la historia intelectual, tomar distancia respecto de dichas denominaciones.

7 Korn es, desde un comienzo, el promotor de los proyectos en los que se nuclea el grupo que lleva adelante la revista, por lo que no es en absoluto extraño que la dirección de la misma quede a su cargo; sin embargo, parece agregarse un elemento más, si consideramos que, en términos conceptuales, son sus definiciones las que le impregnan buena parte de la propuesta editorial.

8 Es probable que Korn use aquí el término "trascendente” como equivalente a "trascendental”. 
9 Recordemos que, en el caso de la UBA, la Facultad de Filosofía y Letras había sido creada a instancias de esta corriente. Si bien sostenemos que es difícil suponer allí ya un campo de saber con reglas definidas que vendrían a disputarse, sí es cierto que, en términos generales, la óptica cientificista tenía mayor legitimidad en la academia porteña.

10 Korn (enero de 1926, tomo 2, p. 11).

11 Destacamos aquí una interesante diferencia con otra publicación de la época: Inicial. Revista de la nueva generación, cuyo estudio y comparación con las que consideramos aquí completaría bastante el panorama de las posiciones reformistas y filosóficas. En relación con lo que venimos planteando, Inicial sí se distancia de la formulación kantiana, y se arriesga en favor del irracionalismo. Sobre este punto, es interesante marcar que el último número de Valoraciones se inicia con un texto de Samuel Ramos que lleva por título "El irracionalismo", y que constituye una dura crítica a esta corriente, en el marco de la cual no se cuida de inscribir a Bergson. Atendiendo una vez más al contexto de publicación, notamos, nos obstante, que ese trabajo va seguido de un "regalo" que la revista hace a sus lectores: "Introducción a la metafísica", de $\mathrm{H}$. Bergson, en la traducción de Carlos Onetti. Al menos queda claro que la revista no se cuida de explicitar las tensiones filosóficas del momento.

12 Esto evoca otra disputa interesante de la época, que gira en torno al relativismo y sus aportes para la filosofía y las disciplinas sociales. Ésta también podría estudiarse cotejando las dos publicaciones que trabajamos aquí.

13 Romero se distanciará de Korn con los años, y será bastante crítico del maestro, en parte introduciendo algunos elementos filosóficos que ya comienzan a reconocerse aquí. Su escasa participación en la revista responde, probablemente, a la brevedad que tenía por entonces su carrera filosófica, y no tanto a esas diferencias (que, repetimos, son posteriores).

14 En marzo del año siguiente, Bermann publica en la Revista de Filosofía una dura crítica a este libro de Ortega, en el que reconoce un cuestionamiento lacerante a la filosofía "naturalista", atenta a los hechos, a la que contrapone el dominio de las ideas. Luego de una extensa argumentación, Bermann termina por condenar la afinidad de esa filosofía con una lógica propia del dogmatismo. Ninguno de los pasajes tomados allí por Bermann, ni de los riesgos marcados, fueron considerados en la reseña de Amaya. Si alguna diferencia presenta allí con el español, parece concentrarse en la centralidad que aquél le da a la masa (que, en ciertos momentos, dada la falta de motivación de ésta, termina por diluir la tarea de los "elegidos").

15 En relación con la vanguardia, cobra singular importancia el apartado destinado al Primer Salón de Escritores, en el número X. Allí se incluye una interesante muestra del arte plástico de vanguardia porteño, en íntimo diálogo con la literatura. Y se explicita el perfil del Salón para el que se convoca. En gesto vanguardista, solicitan a los escritores "una pintura simple, vale decir, pura (¡cavernaria!), lindante con la esquematización geométrica”, y explicita su "intención herética": "quien no se haya bañado alguna vez en las aguas de la herejía, no tiene el espíritu limpio del gran pecado de fanatismo, que es mortal en arte y se llama escuela" ("La redacción”, Valoraciones, 1927, p. 74). Allí se incluyen obras de Girondo, Marechal, Sánchez Reulet, Fatone, Adelina del Carril, Córdoba Iturburu, Marinetti, Borges y Güiraldes, entre otros. Respecto del latinoamericanismo, es importante el texto de Guillermo Korn, "Hacia un arte americano", en que se denuncia el modelo artístico vigente en Occidente, no sólo por desconocer "todo asomo de preocupación trascendental”, sino también porque la consecuencia de ello es la ignorancia de todas las manifestaciones artísticas de "otras razas". "Bajo esta incomprensión asfixiante y espesa hubo de vegetar el arte occidental, y crearse una incomprensiva tolerancia para apreciar las creaciones artísticas de otras razas, valoradas negativamente porque su 'voluntad de forma', no clásica, se apartaba de la copia de la naturaleza”. (Korn, Valoraciones, 1926?, p. 67). Sería sumamente provechoso, para seguir completando el mapa de las posiciones y apuestas, reconocer allí la inscripción de los artistas que se mencionan (Manuel Rodríguez Lozano y Julio Castellanos), en el marco de la vanguardia latinoamericana en general, y de la mexicana en particular, poniéndola en relación con la obra de José Vasconcelos y su política cultural. Sobre Vasconcelos y la Reforma Universitaria, ver Mailhe en este dossier.

16 Luis Rossi reconoce allí un quiebre importante. Creemos, no obstante, que hay muchos elementos que nos permiten reconocer una continuidad en la propuesta de la revista, no obstante esas novedades. Cfr. Rossi (1999).

17 No nos detendremos a analizar cada uno de esos discursos, pero notamos simplemente que resulta muy llamativa la diferencia, en el contenido que se refiere al sentido y el destino de la Universidad, en el caso de Korn, respecto del resto de los decanos. Mientras que allí abunda la idea de un regreso a la posibilidad de postular ideales, con la consecuente condena del positivismo, en los otros casos el perfil universitario es afín a una renovación cientificista de los saberes, puestos al servicio de la modernización del país.

18 Es importante notar que, siendo poco frecuente su participación en la Revista de Filosofía, es, sin embargo, nula en Valoraciones.

19 Recordemos que la noción de "filosofía científica" de Ingenieros fue desarrollada por primera vez en Principios de psicología, de 1911.

20 Orgaz desarrolla estas nociones en varios artículos de la revista, y en ello se observa una interesante proximidad con las ideas de Ingenieros, ideas que atraviesan la publicación desde el texto con el que se abre en 1915, "Para una filosofía argentina”. 
21 Algo muy próximo planteaba, entre otros, el trabajo de Antonio Vidal, "Universidad y filosofía”, publicado en septiembre de 1918. Allí se recuerda, muy cerca de Ingenieros, que la ciencia y la filosofía comparten el método, pero se destaca el valor de la filosofía como orientadora de las actividades personales y colectivas.

22 Es explícita la referencia de Orgaz al modelo que Ingenieros propone en "La filosofía científica en la organización de las universidades”, publicado en el número de marzo de 1916. Pero es importante recordar que Eugenio d'Ors, con argumentos diferentes a los del psiquiatra argentino, sostenía algo similar, y que precisamente la revista se ocupa de ello en la reseña de su texto "El problema de la universidad", publicada en mayo de 1923. Para el catalán, la filosofía era la base de toda carrera universitaria liberal.

23 Algo similar marcan, en otros artículos, Nicolai (1925, pp. 4-6) y Bermann (1924, pp. 161-174), autores también recurrentes en la revista y que claramente comulgan con la línea editorial.

24 Asoma aquí el problema del relativismo, que mencionamos más arriba.

25 Es interesante revisar esa relación compleja con la Universidad. Si bien varios son profesores universitarios, la crítica en general es lapidaria. Pero, además, se preocupan por dejar en claro que el proyecto mismo de la revista no queda para nada atrapado en los márgenes institucionales. Más aún, las revistas de la Universidad son duramente cuestionadas. 